\title{
Evaluation of the Differences between Normal and Cancerous Prostate Tissue Response to Simple and Vibro-Neural Stimulation
}

\author{
Samir Zein ${ }^{\circledR}$, Farhad Tabatabai Ghomsheh $^{2 *}$, Hasan Jamshidian ${ }^{3 ®}$ \\ ${ }^{1}$ Department of Biomedical Engineering, Science and Research Branch, Islamic Azad University, Tehran, Iran \\ ${ }^{2}$ Department of Ergonomics University of Social Welfare and Rehabilitation Sciences, Tehran, Iran. \\ ${ }^{3}$ Department of Urology, Imam-Khomeini Hospital Complex, Tehran University of Medical science, Tehran, Iran.
}

\begin{abstract}
Background: Early detection of prostate cancer has significant benefits for its treatment and can increase the survival chance in patients. In recent years, new methods such as shear wave elastography and vibro-elastography, as well as artificial tactile sensing, have been used to detect a mass in the prostate tissue in-vivo and ex-vivo. This paper aims to investigate the difference between normal and malignant prostate tissue reaction to simple and vibro-neural stimulation for prostate tissue mass detection in order to determine neural stimulation intensity, velocity, and frequency to obtain the best result in detecting the type and location of the tumor.

Methods: This study has utilized neural stimulation devices in normal and cancerous tissues. The stimulation velocity, probe location, and the frequency of neural stimulation considered as the independent variables.

Results: The results show that for superficial masses, although dependent on the probe, the accuracy of detection at the low speed of $5 \mathrm{~mm} / \mathrm{s}$ is $50 \%$ higher than other conditions. On the other hand, in deep masses, with increasing mass depth, the accuracy of detection at the medium speed of $8 \mathrm{~mm} / \mathrm{s}$ is $30 \%$ higher than the low speed. Finally, the results showed that with increased stimulation frequency, the possibility of tumor detection, and its accuracy increases by $35 \%$.

Conclusion: By improving the accuracy of the neural stimulation device, it can apply to detect hard materials such as tumors and malignant tissues.

Keywords: Prostate cancer detection; Neural stimulation; Energy dissipation; Prostate tissue; Residual displacement.
\end{abstract}

*Correspondence to Farhad Tabatabai Ghomsheh, Department of Ergonomics University of Social welfare and Rehabilitation science, Tehran, Iran.

Tel: 98-2122180064; Email: fa.tabatabai@uswr.ac.ir

Published online March 10, 2020

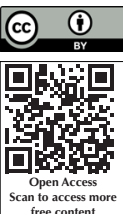

Citation: Zein S, Tabatabai Ghomsheh F, Jamshidian H, Evaluation of the Differences between Normal and Cancerous Prostate Tissue Response to Simple and Vibro-Neural Stimulation. Int Clin Neurosci J. 2020;7(2):61-65. doi:10.34172/icnj.2020.03.

\section{Introduction}

Prostate cancer is the most prevalent type of cancer among men. ${ }^{1}$ According to the US Vital Statistics Center report in $2017,22 \%$ of deaths were due to cancer, with prostate cancer, after lung cancer, as the second leading cause of cancer deaths in men in the United States. ${ }^{1-3}$ Early diagnosis of prostate cancer could have a significant impact on men's health because of early diagnosis, accompanied by proper treatments, enables a 5-year relative survival rate as high as $100 \% .{ }^{1,2}$ Although prostate cancer commonly appears in men who are in their 1960s, the studies showed that one-third of men have histological evidence for prostate adenocarcinoma in their 1940s. ${ }^{4}$ The formation of tumors in prostate tissue is associated with several factors such as age, race, diet, heredity, and environment. ${ }^{5}$

Prostate-specific antigen levels (PSA) in human blood and rectal examination (DRE) are conventional methods of a prostate cancer diagnosis. A prostate biopsy is usually carried out through trans-rectal ultrasound imaging guidance (TRUS). ${ }^{6,7}$ According to the studies on prostate cancer, early biopsies did not lead to prostate cancer diagnosis in a significant number of people with this disease, or due to TRUS error, small tumors that were in the prostate tissue and did not need any treatments diagnosed as malignant tumors. In recent years, new methods such as shear wave elastography, vibro-elastography, vibroacoustography, magnetic resonance, and artificial tactile

(C) 2020 The Author(s). This is an open access article distributed under the terms of the Creative Commons Attribution License (http:// creativecommons.org/licenses/by/4.0/), which permits unrestricted use, distribution, and reproduction in any medium, provided the original work is properly cited. 
techniques have used to detect the presence of the mass in the prostate tissue.

The application of 3D shear wave elastography for clinical prostate cancer detection was proposed by Shoji et ll. $^{8}$ In their research, they used ultrasound pulse pressure to create shear waves in tissues, and they utilized this pulse as a means to measure tissue elasticity, which required trans-rectal guidance of the pulse to the prostate tissue. In this method, sensitivity, specificity, positive prediction, and negative prediction improved by $70 \%$, $98 \%, 91 \%$, and $92 \%$ in prostate tissue, respectively, and prostate lesions elasticity significantly correlated with Gleason score. ${ }^{8}$ Alizad et al and Mitri et al examined the application of vibro-acoustography in prostate tissue imaging and pointed out that the VA may use in clinical in-vivo prostate imaging. ${ }^{9,10}$

There are also several neural simulations studies ${ }^{11-20}$; however, artificial tactile sense and its applications widely used for mass detection. Using artificial tactile, Peng et al measured the stiffness of different prostate tissues and concluded that this method is capable of reporting the stiffness of different tissues. ${ }^{21}$ A robotic system with sweeping palpation and needle biopsy was invented by Ahn et al to detect prostate cancer. ${ }^{22,23}$ The system designed to be capable of simultaneous tactile examination and biopsy. The results of ex-Vitro studies on human prostate and artificial tissue showed that this method had $81 \%$ accuracy in the stiff mass detection..$^{22,23}$

Given that the formation of cancerous masses is a sign of excessive reproduction of the cells, and that based on the conducted studies, most cancerous masses formed in the peripheral zone, accumulation of cells in the prostate tissue increases the number of blood vessels in the prostate tissue, which in turn results in the accumulation of nerves in the given area. ${ }^{24,25}$ Therefore, neural stimulation of the prostate tissue and the tissue response to the stimulation can be an alternative method for standard clinical examinations that are unable to detect tumors in early stages. This study examines the difference between normal and cancerous prostate tissue response to the simple and vibro-neural stimulation. In all previous studies, all mass detection in the prostate tissue was done through the rectum and via elastography and artificial tactile methods, whereas this study aims to detect the presence of a mass in the prostate tissue through the perineum with neural stimulation

\section{Materials and Methods \\ Study Design}

Simple and Neural stimulation on normal and cancerous prostate tissue and the reaction of the tissues used to investigate the presence or absence of the mass. Since the prostate tissue is symmetrical and the characteristics of forming cancerous masses are similar to the prostate tissue, ${ }^{26}$ to analyze the neural stimulation velocity and frequency on the tissue, neural stimulation applied to the prostate gland at 0,5 , and $10 \mathrm{~Hz}$ with different velocities. Once again, it was placed on the spherical zone of the prostate gland; and the conditions of the previous states repeated. Each test was conducted three times with 10 -minute intervals. The test results analyzed by SPSS 16 software and the mathematical relationships governing the soft tissue obtained as the output

\section{The Device Design}

The instrument used for conducting experiments on tissues is Neural Stimulation Device (NSD) (Figure 1). Bending load cell (MBL FUTEK, USA), DC motor (Eziservo FASTECH, Korea), pressure sensor (MBL), encoder (HP, USA), micro switch, and probe are the components of the instrument. It designed for $1 \mathrm{~cm}$ displacement and loading velocities of 5,8 , and $11 \mathrm{~mm} / \mathrm{s}$ at 0,5 , and $10 \mathrm{~Hz}$ frequencies. Standard methods calibrate it.

\section{Study Population}

The study prospectively recruited 5 patients with serum PSA levels of 5.0-18.0 ng/mL who suspected of having prostate cancer and 5 volunteers without any prostate diseases. All subjects gave their informed consent for inclusion before participating in the study.

\section{Result and Discussion}

This study used a neural stimulation system to apply vibrational and straightforward stimulation with five and $10 \mathrm{~Hz}$ frequencies on normal and cancerous prostate tissues and studied the different responses of the 2 tissues. The maximum force imposed by the prostate tissue to the device probe, the residual displacement after neural stimulation, as well as the amount of energy dissipation in the tissue investigated as the main variables in the

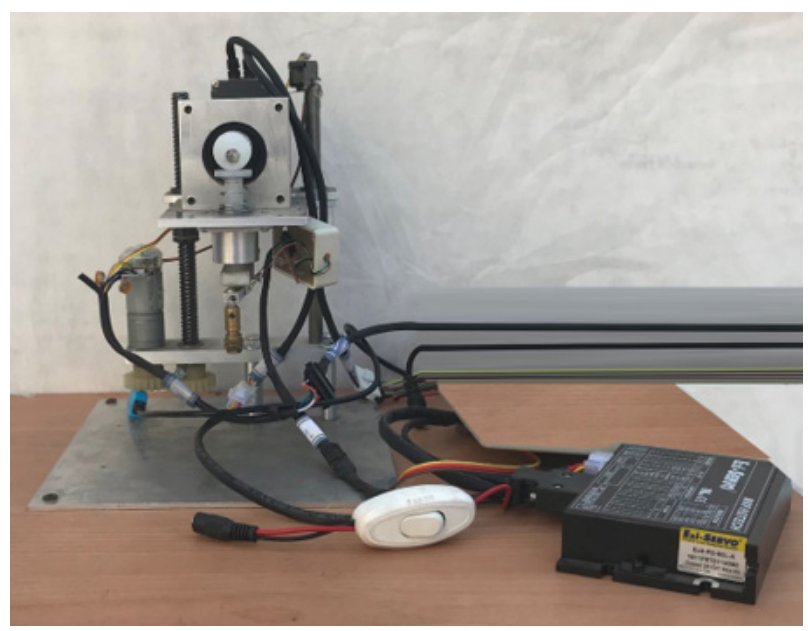

Figure 1. NSD and its Components for Applying Simple and Vibro-neural Stimulation on the Tissue. 
feasibility study of the cancerous mass detection. Also, the probe location for applying neural stimulation was studied as a parameter to enhance mass detection accuracy.

\section{The Effect of Maximum Force Applied to the Probe}

The results showed that the maximum force applied to the probe by the cancerous tissue is higher than the force applied the normal tissue in both vibrational and straightforward stimulation because of the cancerous tissue has larger elastic modulus due to the tumors in it (Figures 2 and 3).

The comparison of force-displacement figures in the cancerous tissue showed that by amplifying the stimulation frequency from 0 to $10 \mathrm{~Hz}$, the diagram slope increases, indicating that enhanced frequency of neural stimulation would increase mass detection possibility by $20 \%$ in the prostate tissue. By displacing the probe from CZ (Central Zone) to PZ ( Peripheral Zone), the accuracy of the cancerous mass detection increased by $15 \%$.

\section{The Effect of the Stimulation Velocity}

Regarding the fact that the prostate tissue is viscoelastic, and that in viscoelastic materials, stiffness is a function of stimulation velocity, the result showed that in case of surface masses, with increased velocity of simple

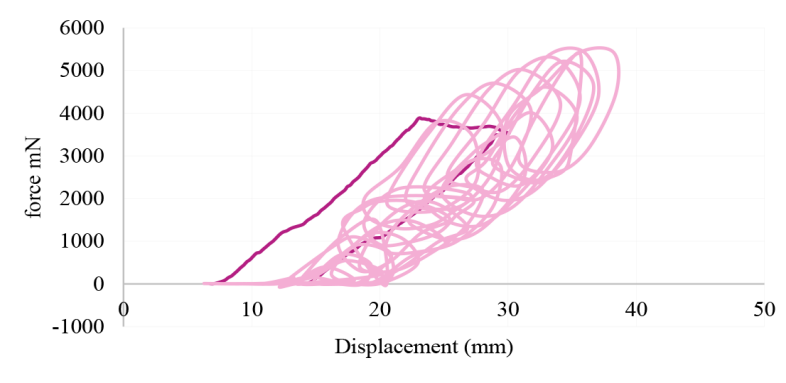

- simple stimulation $\quad$ vibro stimulation

Figure 2. . Force-Displacement Curves of Simple and Vibroneural Stimulation for the Cancerous Tissue at $5 \mathrm{~mm} / \mathrm{s}$.

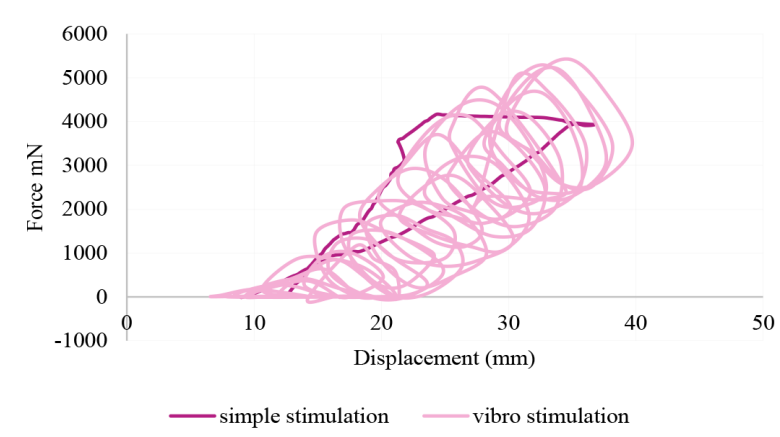

Figure 3. Force-Displacement Curves of Simple and Vibro-neural Stimulation for the Normal Tissue at $5 \mathrm{~mm} / \mathrm{s}$. stimulation, the stiffness difference of normal and cancerous tissues decreased from $14 \%$ to $8 \%$ and from $8 \%$ to $4 \%$, respectively. As a result, the most optimal velocity is $5 \mathrm{~mm} / \mathrm{s}$, which enhances mass detection accuracy by $14 \%$ compared to other states.

In the case of deep masses, simple neural stimulation is not capable of mass detection. Thus, vibro-neural stimulation was used. The result showed that by amplifying the speed from $5 \mathrm{~mm} / \mathrm{s}$ to $8 \mathrm{~mm} / \mathrm{s}$, the possibility of mass detection increased by $40 \%$. By increasing the frequency from 5 to $10 \mathrm{~Hz}$ in vibrational stimulation at $5 \mathrm{~mm} / \mathrm{s}, 8$ $\mathrm{mm} / \mathrm{s}$, and $11 \mathrm{~mm} / \mathrm{s}$ velocities, the detection accuracy increased by $12 \%, 22 \%$, and $13 \%$, respectively.

\section{The Effect of Residual Displacement}

Since the prostate tissue is a viscoelastic material and has the properties of elastic and viscous materials simultaneously, the loading and unloading curves do not overlap, and as a result, a residual strain always remains in such materials. Given that residual displacement represents residual strain in the force-displacement graph, at the same stimulation velocity and in different probe locations, residual displacement in the cancerous tissue is perceived to be lower than that of the normal tissue, indicating that the viscosity of the cancerous tissue is less than the normal one. It has also observed that with increased stimulation velocity, the amount of residual displacement in the cancerous tissue has increased from $3.8 \%$ to $7.9 \%$ and from $7.9 \%$ to $8.38 \%$, which means that with increased stimulation velocity, the viscosity of the phantom enhances as well. Consequently, it is more likely that the mass can detect at a lower velocity (Figure 4).

In vibro-neural stimulation, it has observed that the value of residual displacement in the normal tissue is higher than the cancerous tissue, and it has observed that the highest percentage of difference between the two types of the tissues is at the medium velocity (26\%), and when the velocity increases to $11 \mathrm{~mm} / \mathrm{s}$, the probability of detection error increases. Although at $8 \mathrm{~mm} / \mathrm{s}$ velocity

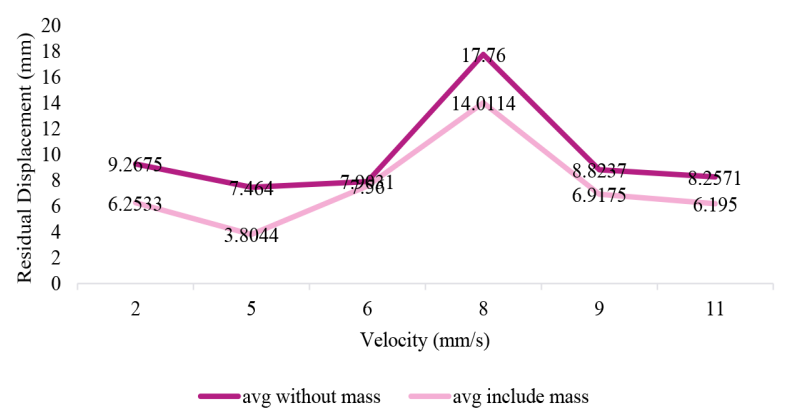

Figure 4. Residual Displacement of Normal and Cancerous Tissues at Simple Stimulation at Different Velocities and Probe Locations. Vertical and horizontal axes show residual displacement $(\mathrm{mm})$ and velocity $(\mathrm{mm} / \mathrm{s})$, respectively. 
mass detection is independent of the probe location, the accuracy of mass detection in $\mathrm{PZ}$ probing is $23 \%$ higher than CZ probing (Figure 5). The amount of residual displacement is one of the features that can be considered equivalent to the physician's tactile sense when touching the tissue and to the degree of tissue deformability

\section{The Effect of Energy Dissipation}

The area under the force-displacement curve (F-D) represents energy dissipation. Regarding measuring the area under these curves and comparing them, it has concluded that the area under the cancerous tissue is less than the normal tissue, indicating that the normal tissue has more viscosity than the cancerous tissue, which means it has a lower elastic modulus. The results of the area under force-displacement graph showed that at the same simple stimulation velocity and varied probe location, the percentage of energy dissipation difference is more significant in $\mathrm{PZ}$, and with increased stimulation

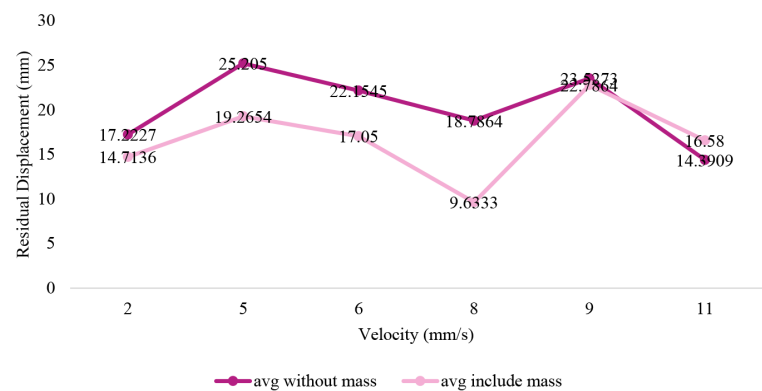

Figure 5. Residual Displacement of Normal and Cancerous Tissues in Vibro-neural stimulation at $10 \mathrm{~Hz}$ at Different Velocities and Probe Locations. Vertical and horizontal axes show residual displacement $(\mathrm{mm})$ and velocity $(\mathrm{mm} / \mathrm{s})$, respectively.

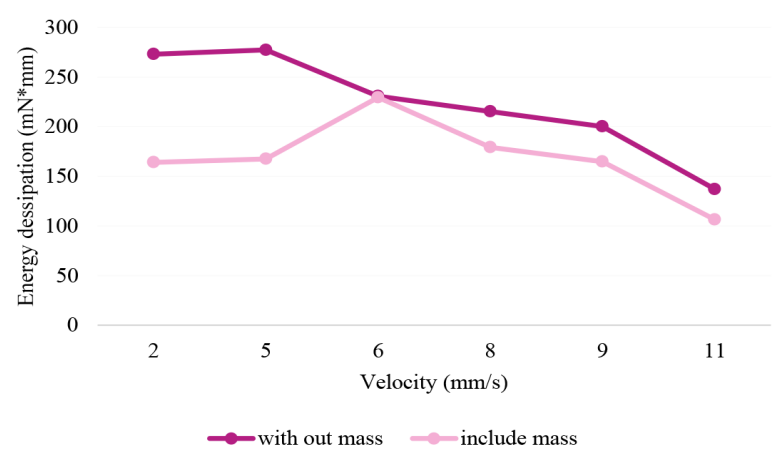

Figure 6. Energy Dissipation of Normal and Cancerous Tissues in Simple Stimulation at Different Velocities and Probe Location. Vertebral and horizontal axes show energy dissipation and velocity $(\mathrm{mm} / \mathrm{s})$, respectively.

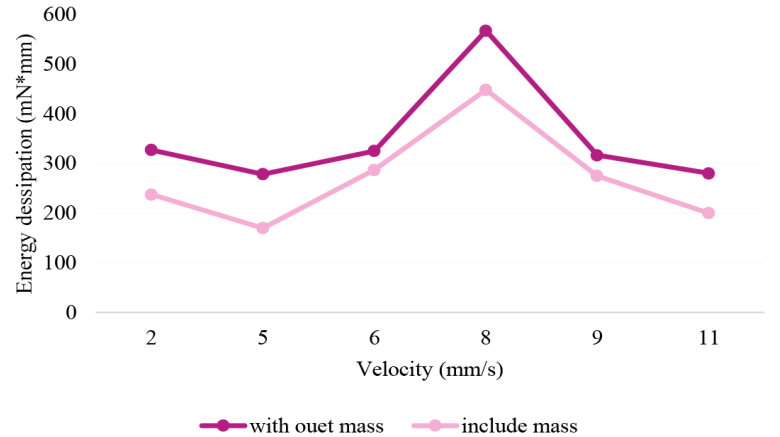

Figure 7.Energy Dissipation of Normal and Cancerous Tissues in Vibro-neural Stimulation at Different Velocities and Probe Locations. Vertebral and horizontal axes show energy dissipation and velocity $(\mathrm{mm} / \mathrm{s})$, respectively.

velocity in both probing states, the percentage of energy dissipation difference is reduced by $17 \%, 9 \%$ respectively, indicating that low velocity is more suitable in detecting surface masses (Figure 6).

In vibrational stimulation, it has observed that at the speed of $8 \mathrm{~mm} / \mathrm{s}$, the difference between the energy dissipation in the massed and non-massed tissue with different probe locations was approximately the same, and the energy dissipation was $20 \%$ and $24 \%$ higher than the $5 \mathrm{~mm} / \mathrm{s}$ and $11 \mathrm{~mm} / \mathrm{s}$ velocities, respectively (Figure 7).

\section{Conclusion}

This study took a new step in measuring tissue stiffness concerning the force the tissue applies to the probe, and because stiffness varies in normal and mass-containing tissues, this method can be accompanied by biopsies to increase the detection power. Vibrational stimulation is not required for surface mass detection, and low stimulation velocity can detect by this method, but to detect deep masses. It is necessary to increase the stimulation velocity and to create vibration. Future studies will examine the effect of increased frequency of the applied neural stimulation as well as decreased noise in the test process. By improving the accuracy of the neural stimulation device, this system can apply to detect hard materials such as tumors and malignant tissues.

\section{Authors' contributions}

All the authors contributed substantially to the conception and design of this study. S Zein contributed substantially to the acquisition of data. S Zein and F Tabatabai Ghomsheh analyzed and interpreted the data. All the authors drafted the manuscript. $\mathrm{H}$ Jamshidian and S Zein contributed substantially to its critical revision. All the authors approved the final version submitted for publication and take responsibility for the statements made in the published article. 


\section{Conflict of Interest Disclosures}

The authors declare that they have no conflict of interests.

\section{Ethical Statement}

All procedures performed in the studies were under the ethical standards of the University of Social Welfare and Rehabilitation Sciences and the 1964 Helsinki Declaration and its later amendments or comparable ethical standards. Furthermore, this article does not contain any studies with animals performed by any of the authors. In this study, informed consent obtained from all participants and confidentiality of the information emphasized.

\section{References}

1. Murphy SL, Xu J, Kochanek KD, Arias E. Mortality in the United States, 2017. NCHS Data Brief. 2018(328):1-8.

2. Heron M. Deaths: leading causes for 2016. Natl Vital Stat Rep. 2018;67(6):1-77.

3. Åstrand AP, Andersson BM, Jalkanen V, Ljungberg B, Bergh A, Lindahl OA. Prostate cancer detection with a tactile resonance sensor--measurement considerations and clinical setup. Sensors (Basel). 2017;17(11). doi: 10.3390/s17112453.

4. Sakr WA, Grignon DJ, Crissman JD, Heilbrun LK, Cassin BJ, Pontes JJ, et al. High grade prostatic intraepithelial neoplasia (HGPIN) and prostatic adenocarcinoma between the ages of 20-69: an autopsy study of 249 cases. In Vivo. 1994;8(3):43943.

5. Karan D, Thrasher JB, Lubaroff D. Prostate cancer: genes, environment, immunity and the use of immunotherapy. Prostate Cancer Prostatic Dis. 2008;11(3):230-6. doi: 10.1038/pcan.2008.3.

6. Woo S, Kim SY, Cho JY, Kim SH. Shear wave elastography for detection of prostate cancer: a preliminary study. Korean J Radiol. 2014;15(3):346-55. doi: 10.3348/kjr.2014.15.3.346.

7. Woo S, Suh CH, Kim SY, Cho JY, Kim SH. Shear-wave elastography for detection of prostate cancer: a systematic review and diagnostic meta-analysis. AJR Am J Roentgenol. 2017;209(4):806-14. doi: 10.2214/ajr.17.18056.

8. Shoji S, Hashimoto A, Nakamura T, Hiraiwa S, Sato H, Sato $Y$, et al. Novel application of three-dimensional shear wave elastography in the detection of clinically significant prostate cancer. Biomed Rep. 2018;8(4):373-7. doi: 10.3892/ br.2018.1059.

9. Alizad A, Mehrmohammadi M, Mitri FG, Davis BJ, Sebo TJ, Mynderse LA, et al. Application of vibro-acoustography in prostate tissue imaging. Med Phys. 2013;40(2):022902. doi: 10.1118/1.4773890

10. Mitri FG, Urban MW, Fatemi M, Greenleaf JF. Combined Vibro-acoustography (VA) imaging and Shearwave Dispersion Ultrasonic Vibrometry (SDUV) for measuring prostate viscoelastic material properties - An in vitro feasibility study. Proceedings of 20th International Congress on Acoustics, ICA; Sydney, Australia; 2010.

11. Gholampour S. FSI simulation of CSF hydrodynamic changes in a large population of non-communicating hydrocephalus patients during treatment process with regard to their clinical symptoms. PLoS One. 2018;13(4):e0196216. doi: 10.1371/ journal.pone.0196216.

12. Gholampour S, Bahmani M, Shariati A. Comparing the efficiency of two treatment methods of hydrocephalus: shunt implantation and endoscopic third ventriculostomy. Basic Clin Neurosci. 2019;10(3):185-98. doi: 10.32598/bcn.9.10.285.

13. Gholampour $\mathrm{S}$, Deh $\mathrm{HHH}$. The effect of spatial distances between holes and time delays between bone drillings based on examination of heat accumulation and risk of bone thermal necrosis. Biomed Eng Online. 2019;18(1):65. doi: 10.1186/ s12938-019-0686-6.

14. Gholampour S, Fatouraee N, Seddighi AS, Seddighi A. Numerical simulation of cerebrospinal fluid hydrodynamics in the healing process of hydrocephalus patients. J Appl Mech Tech Phys. 2017;58(3):386-91. doi: 10.1134/ s0021894417030026.

15. Gholampour S, Fatouraee N, Seddighi AS, Seddighi A. Evaluating the effect of hydrocephalus cause on the manner of changes in the effective parameters and clinical symptoms of the disease. J Clin Neurosci. 2017;35:50-5. doi: 10.1016/j. jocn.2016.09.012.

16. Gholampour S, Hajirayat K. Minimizing thermal damage to vascular nerves while drilling of calcified plaque. BMC Res Notes. 2019;12(1):338. doi: 10.1186/s13104-019-4381-2.

17. Gholampour S, Jalali A. Thermal analysis of the dentine tubule under hot and cold stimuli using fluid-structure interaction simulation. Biomech Model Mechanobiol. 2018;17(6):1599610. doi: 10.1007/s10237-018-1046-3.

18. Gholampour S, Taher M. Relationship of morphologic changes in the brain and spinal cord and disease symptoms with cerebrospinal fluid hydrodynamic changes in patients with Chiari malformation type I. World Neurosurg. 2018;116:e830-e9. doi: 10.1016/j.wneu.2018.05.108.

19. Hajirayat K, Gholampour S, Sharifi I, Bizari D. Biomechanical simulation to compare the blood hemodynamics and cerebral aneurysm rupture risk in patients with different aneurysm necks. J Appl Mech Tech Phys. 2017;58(6):968-74. doi: 10.1134/s0021894417060025.

20. Taher M, Gholampour S. Effect of ambient temperature changes on blood flow in anterior cerebral artery of patients with skull prosthesis. World Neurosurg. 2019. doi: 10.1016/j. wneu.2019.11.171.

21. Peng Q, Omata S, Peehl DM, Constantinou CE. Stiffness mapping prostate biopsy samples using a tactile sensor. Conf Proc IEEE Eng Med Biol Soc. 2011;2011:8515-8. doi: 10.1109/iembs.2011.6092101.

22. Ahn B, Kim Y, Oh CK, Kim J. Robotic palpation and mechanical property characterization for abnormal tissue localization. Med Biol Eng Comput. 2012;50(9):961-71. doi: 10.1007/s11517-012-0936-2.

23. Ahn B, Lee $H, \operatorname{Kim} Y$, Kim J. Robotic system with sweeping palpation and needle biopsy for prostate cancer diagnosis. Int J Med Robot. 2014;10(3):356-67. doi: 10.1002/rcs.1543.

24. Sciarra A, Mariotti G, Salciccia S, Autran Gomez A, Monti S, Toscano V, et al. Prostate growth and inflammation. J Steroid Biochem Mol Biol. 2008;108(3-5):254-60. doi: 10.1016/j. jsbmb.2007.09.013.

25. Vasto S, Carruba G, Candore G, Italiano E, Di Bona D, Caruso C. Inflammation and prostate cancer. Future Oncol. 2008;4(5):637-45. doi: 10.2217/14796694.4.5.637.

26. Liau J, Goldberg D, Arif-Tiwari H. Prostate Cancer Detection and Diagnosis: Role of Ultrasound with MRI Correlates. Curr Radiol Rep. 2019;7(3):7. doi: 10.1007/s40134-019-0318-8. 